\title{
Utility of 123I-iomazenil single-photon emission computed tomography as an indicator of neurological irreversibility in patients with non-convulsive status epilepticus
}

\author{
Miwa $\mathrm{K}^{1 *}$, Ohmura $\mathrm{K}^{1}$, Yoshimura $\mathrm{K}^{1}$, Shinoda $\mathrm{J}^{2}$, Nakayama $\mathrm{N}^{3}$, Yano $\mathrm{H}^{3}$ and Iwama $\mathrm{T}^{3}$ \\ ${ }^{1}$ Department of Neurosurgery, Gifu Prefectural Gero Hospital, Gero, Gifu, Japan \\ ${ }^{2}$ Chubu Medical Center for Prolonged Traumatic Brain Dysfunction, Kizawa Memorial Hospital, Minokamo, Gifu, Japan \\ ${ }^{3}$ Department of Neurosurgery, Gifu University Graduate School of Medicine, Gifu, Japan
}

\begin{abstract}
We describe two cases of impaired consciousness due to NCSE which were evaluated using IMZ-SPECT. In the first case, IMZ-SPECT revealed a sparse and mild decrease in IMZ uptake. In the other case, IMZ-SPECT revealed an extensive and marked decrease in IMZ uptake. The first patient recovered from impaired consciousness, whereas the other demonstrated continued stupor and died of pneumonia. Assessment using IMZ-SPECT might be useful for prediction of neurological prognoses in patients with NCSE.
\end{abstract}

Abbreviations: NCSE: Non-Convulsive status epilepticus; EEG: Electroencephalography; IMZ-SPECT: 123I-iomazenil SPECT; IMPSPECT: N-isopropyl-p-[I123] iodoamphetamine SPECT.

\section{Introduction}

Non-convulsive status epilepticus (NCSE) is diagnosed in practice as an enduring epileptic condition with reduced or altered consciousness, but without major convulsive movements, [1] and with epileptiform discharges on electroencephalography (EEG). Peri- and postictal changes detected by both anatomic and functional imaging have been recognized for many years [2]. With the wide availability of MRI and SPECT, a growing range of acute imaging findings have been described. While some of these findings have been shown to be reversible, it is not fully understood if they can also detect the reversibility of neuronal tissue damage in the epileptogenic zone in patients with NCSE.

Herein, we report two cases of NCSE with unknown etiology that satisfied the Salzburg Criteria. Evaluation by 123I-iomazenil SPECT (IMZ-SPECT) showed different patterns of tracer uptake in the presumed epileptogenic zone: extensively and markedly decreased IMZ uptake compared to sparsely and mildly decreased IMZ uptake and discuss the clinical and therapeutic significance [3]. We also evaluated N-isopropyl-p-[I123] iodoamphetamine SPECT (IMPSPECT), DWI, and clinical outcomes in these patients. Based on results from follow-up MRI and the presence of neurological sequelae, we diagnosed irreversible neuronal damage in which IMZ-SPECT showed an extensive and marked decrease in IMZ uptake in the corresponding regions. Lastly, we investigated the clinical utility of IMZ-SPECT as a complementary modality for predicting neuropathological prognoses in cases of NCSE.

\section{Case reports}

Case 1: An 82-year-old woman developed sudden generalized tonic-clonic seizure. There was neither medical history nor family history. Laboratory examinations, including blood and urine routine examination, hematological, biochemistry, coagulation profile, thyroid function, cerebrospinal fluid routine, cerebrospinal fluid immunoantibodies, and tumor biomarkers were all normal.

Although generalized seizure attack was alleviated after the administration of diazepam, she remained in a reduced consciousness. EEG showed high frequency of epileptiform discharges, satisfying the diagnosis of NCSE (Figure 1A). In the acute phase, IMP-SPECT showed high levels of tracer accumulation in the left temporal lobe (Figure 1B), and DWI showed an area of abnormally high signal intensity in the left temporal lobe (Figure 1C). Original image of IMZ-SPECT showed no remarkable abnormality, although stereotactic surface projection analysis showed sparsely and mildly decreased uptake of IMZ in the left temporal lobe (Figure 1D). $1000 \mathrm{mg}$ of levetiracetam was started. The patient recovered from a reduced consciousness within several days; however, mild memory disturbance remained. The patient received follow-up MRI and EEG one month after seizure onset. FLAIR showed no abnormality in the left temporal lobe (Figure 1E). EEG showed no continued epileptic discharges. She was subsequently discharged and living healthily with some assistance.

${ }^{*}$ Correspondence to: Kazuhiro Miwa, MD, PhD, Department of Neurosurgery, Gifu Prefectural Gero Hospital, Gero, Gifu, Japan, 2211 Mori-cho, Gero, Gifu 509-2292, Japan, E-mail: doctor.3@jasmine.ocn.ne.jp

Received: January 14, 2019; Accepted: February 01, 2019; Published: February 05, 2019 
Miwa K (2019) Utility of 123I-iomazenil single-photon emission computed tomography as an indicator of neurological irreversibility in patients with non-convulsive status epilepticus

Case 2: A 73-year-old man developed sudden generalized tonicclonic seizure. There was neither medical history nor family history, and laboratory examinations were all normal. Although generalized seizure attack was alleviated after the infusion of diazepam, he remained in a comatose state with subtle clinical ictal phenomena such as epileptic nystagmus and mild myoclonus. EEG showed periodic epileptiform discharges with subtle clinical ictal phenomena, satisfying the diagnosis of NCSE (Figure 2A). During the acute phase, IMP-SPECT showed high levels of tracer accumulation in the left temporo-occipital lobes (Figure 2B), and DWI showed areas of abnormally high signal intensity in the left temporo-occipital lobes

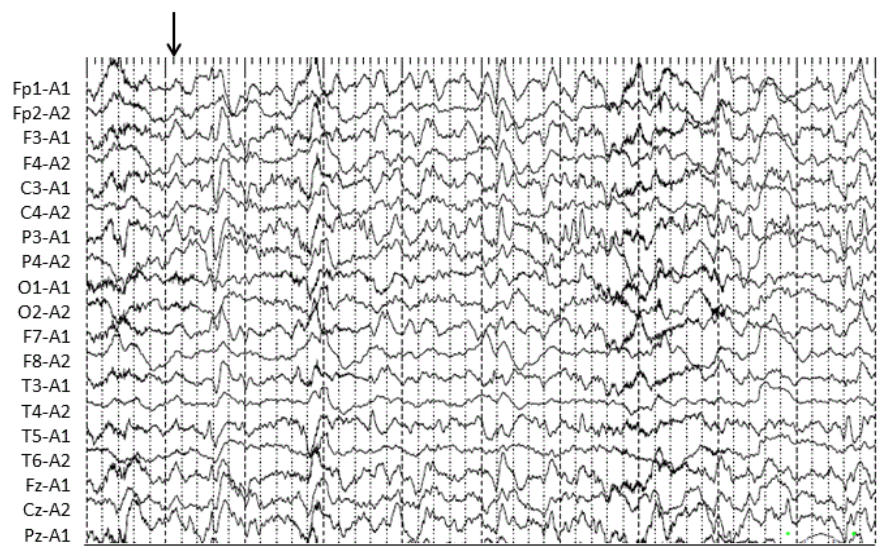

Figure 1A. EEG performed at the onset of seizure showed high frequency of epileptiform discharges, satisfying the diagnosis of NCSE. An arrow indicates the onset of the seizure, which seems to be located mainly in the left parietal areas

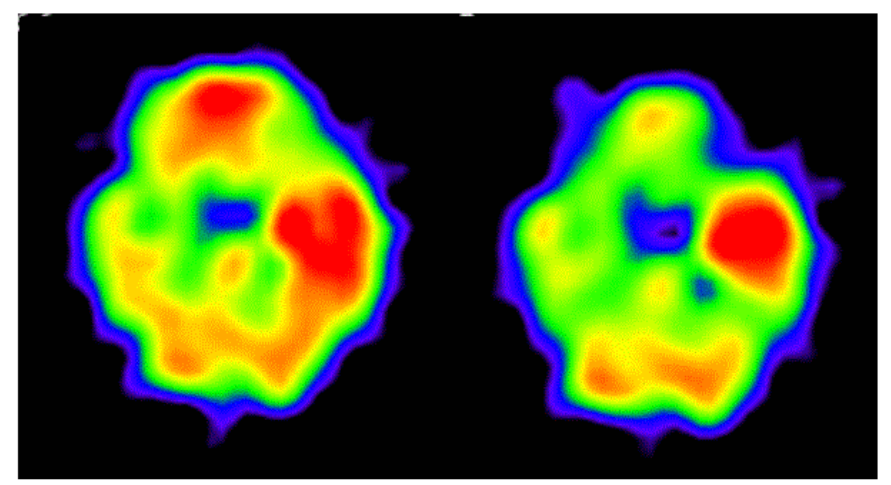

Figure 1B. In the acute phase, IMP-SPECT showed high levels of tracer accumulation in the left temporal lobe, which was $25 \%$ higher than in the contralateral lobe

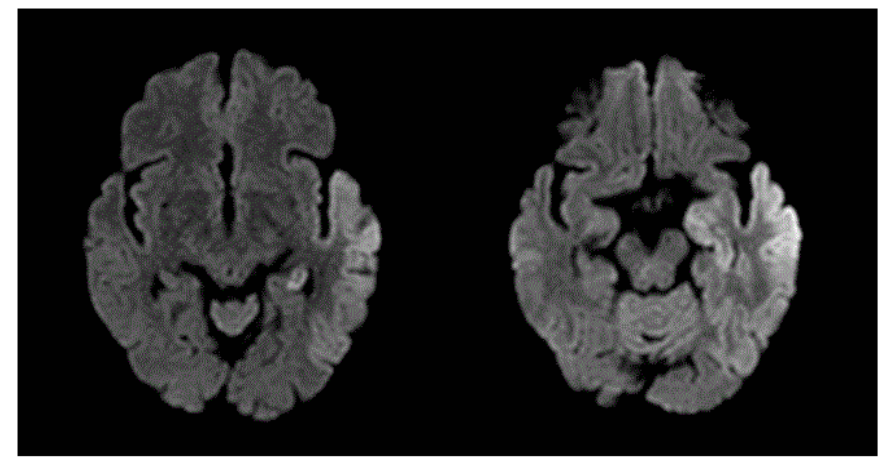

Figure 1C. In the acute phase, DWI showed areas of abnormally high signal intensity along the gyri in the left temporal lobe

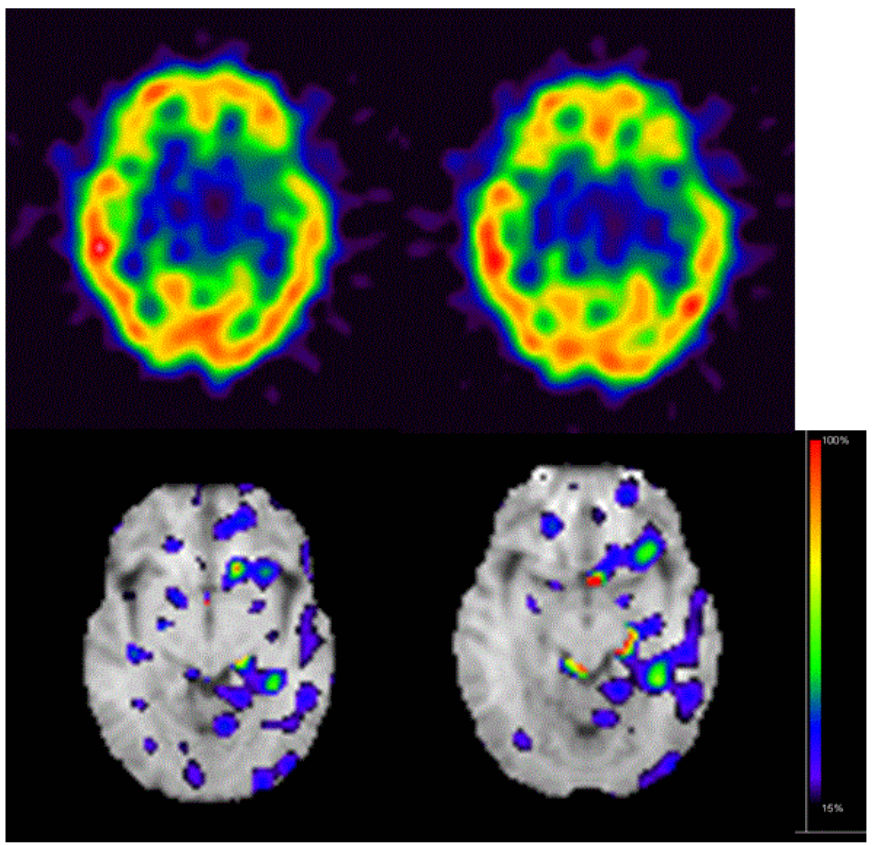

Figure 1D. Original image of IMZ-SPECT showed no remarkable abnormality in the left temporal lobe (upper row). Stereotactic surface projection analysis of IMZ-SPECT showed mostly normal uptake in the left temporal lobe, although there was mildly decreased uptake with abnormal findings restricted to a small portion of the neocortex (lower row). The color bar indicates the Z-score of the decrease in IMZ uptake, with red indicating the strongest decrease in IMZ uptake

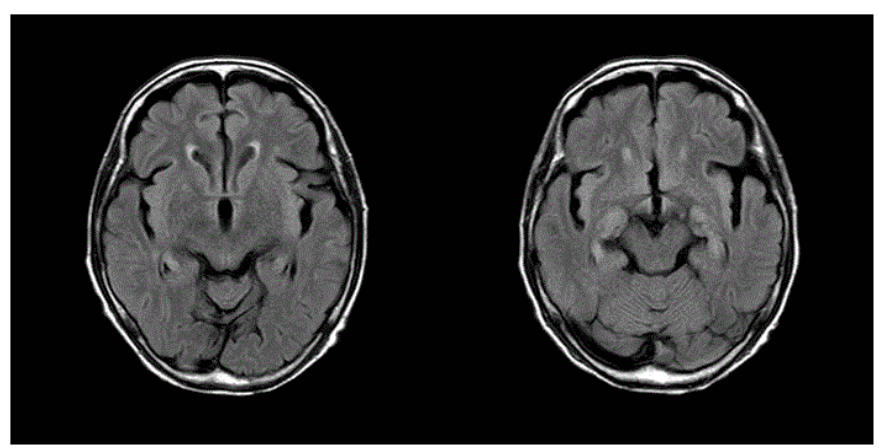

Figure 1E. FLAIR imaging one month after seizure onset showed normal signal intensity in the left temporal lobe, in which high signal intensity had been detected by DWI during the acute phase

(Figure 2C). Original image and stereotactic surface projection analysis showed an extensive and marked decrease in IMZ uptake in the left temporo-occipital lobes (Figure 2D).

In spite of intense antiepileptic treatment with $3000 \mathrm{mg}$ of levetiracetam and $200 \mathrm{mg}$ of lacosamide, the patient failed to recover from a comatose state. The patient received follow-up MRI and EEG one month after seizure onset. FLAIR showed high signal intensity in the left temporo-occipital lobes, suggesting cortical laminar damage (Figure 2E). EEG showed localized slowing in the left temporo-occipital areas. The patient experienced intractable aspiration pneumonia and died 37 days after seizure onset.

\section{Discussion}

While IMZ is a relatively new radioactive tracer that has been applied to SPECT for the study of brain activity, it has also been shown to be useful for identifying pathological regions in patients 
Miwa K (2019) Utility of 123I-iomazenil single-photon emission computed tomography as an indicator of neurological irreversibility in patients with non-convulsive status epilepticus

with temporal lobe epilepsy. One of the advantages of IMZ-SPECT is its selectivity for central type gamma-aminobutyric acid-A $\left(\mathrm{GABA}_{\mathrm{A}}\right) /$ benzodiazepine receptors [4-6]. Since GABA is the major inhibitory neurotransmitter in the brain, IMZ-SPECT enables the detection of GABA activity, which provides representative pharmacodynamic imaging of the central nervous system [7-9].

At baseline, GABA inhibitory neurotransmission predominates over N-methyl-D-aspartate (NMDA) excitatory neurotransmission. During seizure, GABA receptors are internalized, while NMDA receptors accumulate in the postsynaptic membrane. These changes result in self-sustaining seizures and resistance to antiepileptic drugs, such as benzodiazepines, via a GABAergic mechanism. In cases of decreased IMZ uptake, under-expression of GABA receptors could

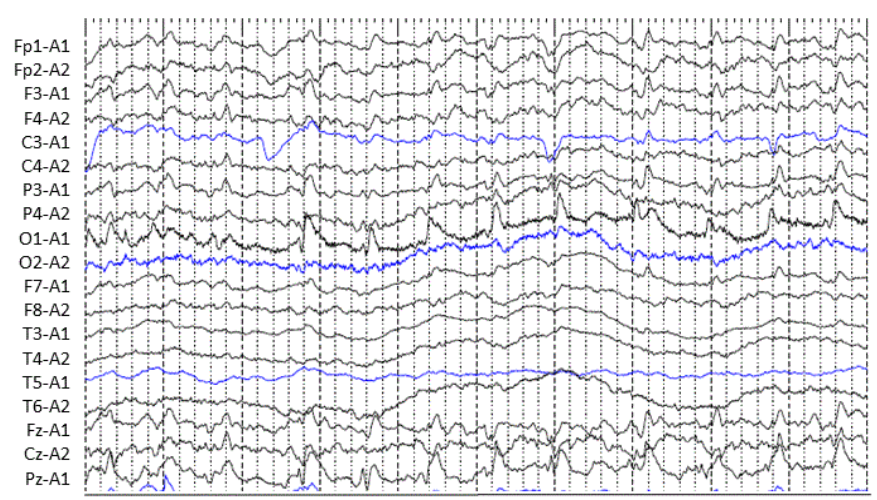

Figure 2A. EEG performed at the onset of seizure showed periodic lateralized epileptiform discharges. In the continuous EEG recordings, Case 2 showed subtle clinical icta phenomena such as epileptic nystagmus and mild myoclonus, satisfying the diagnosis of NCSE

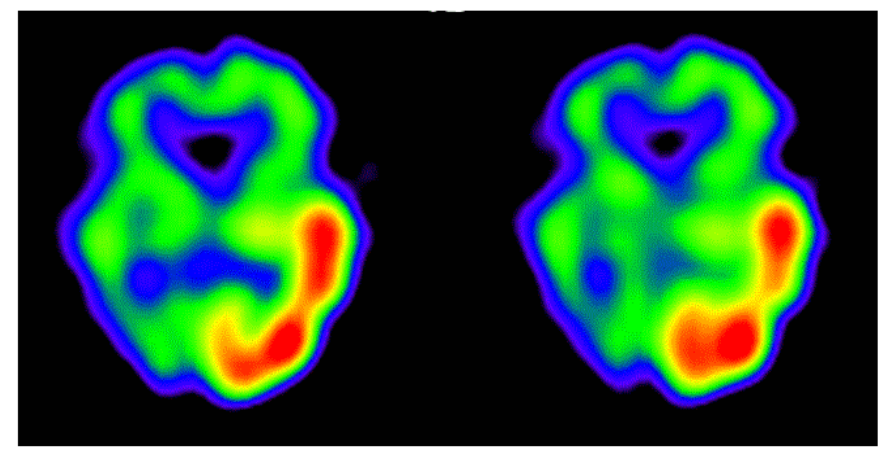

Figure 2B. In the acute phase, IMP-SPECT showed high levels of tracer accumulation in the left temporo-occipital lobes, which were $40 \%$ higher than in the contralateral lobe

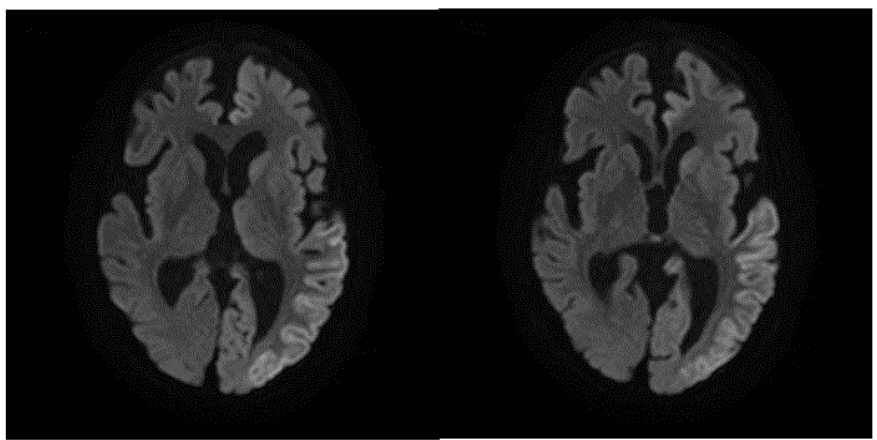

Figure 2C. In the acute phase, DWI showed areas of abnormally high signal intensity along the gyri in the left temporo-occipital lobes

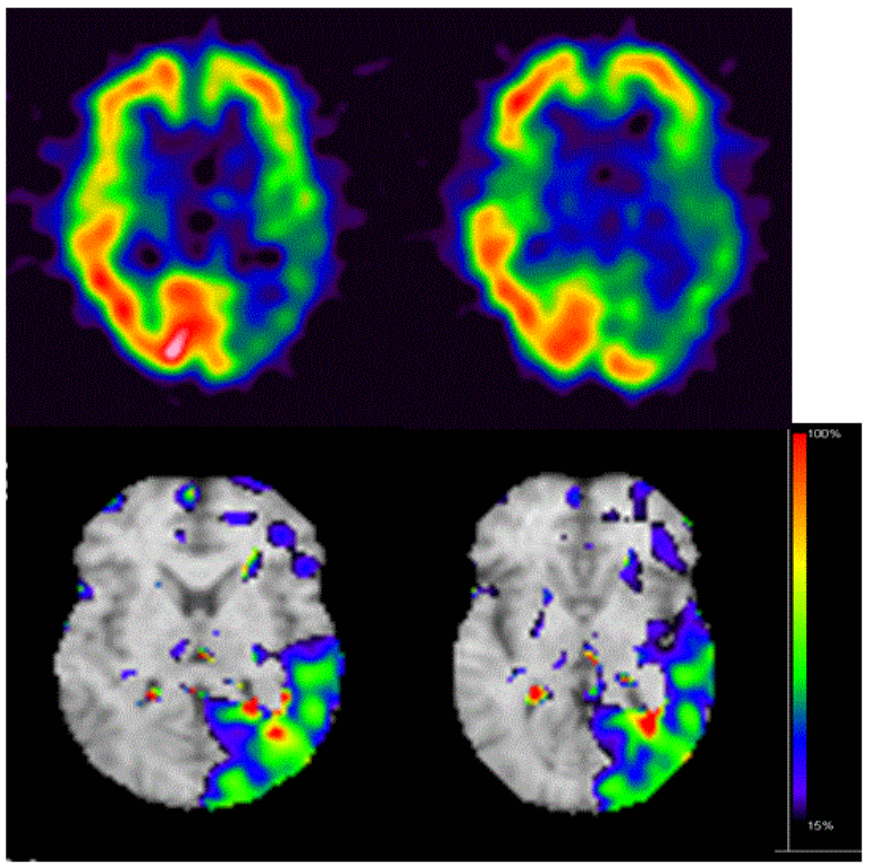

Figure 2D. Original image of IMZ-SPECT showed marked decrease of tracer accumulation in the left temporo-occipital lobes (upper row). Stereotactic surface projection analysis of IMZ-SPECT showed remarkably decreased IMZ uptake extensively throughout the left temporo-occipital lobes (lower row). The color bar indicates the Z-score similar to that in Figure 1

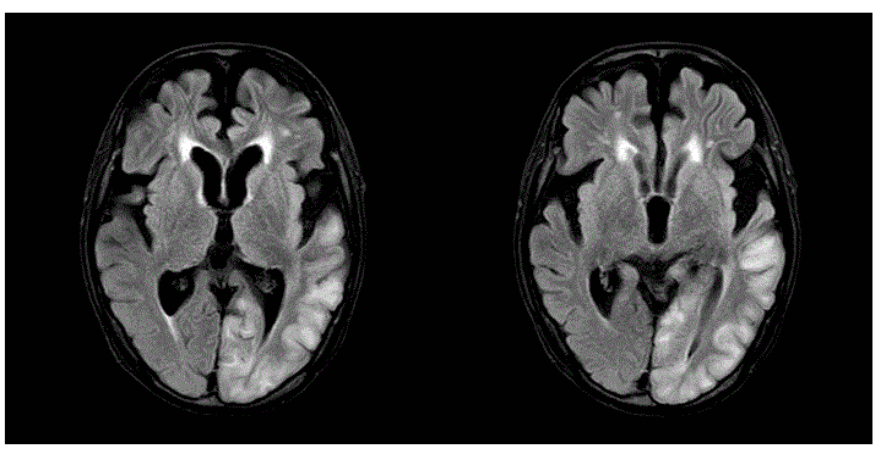

Figure 2E. FLAIR imaging one month after seizure onset showed high signal intensity in the subcortical region of the left temporo-occipital lobes, suggesting cortical laminar damage

result in intractable and irreversible neuropathological degeneration [10].

In view of this neuropathological irreversibility, Bauer et al. depicted the various forms of NCSE along a three-dimensional biological continuum. The first axis represents the gradual impairment of consciousness [2]. The second axis represents the degree of structural brain damage; this axis largely determines the prognosis. The third axis represents the degree to which the ongoing epileptic activity contributes to the depth of coma and additive structural brain damage due to excessive epileptic activity. In the present study, the case with a sparse and mild decrease in IMZ uptake could largely be attributed to epileptic brain activity (Figure 1D). In contrast, the case with an extensive and marked decrease in IMZ uptake might be attributed to structural brain damage (Figure 2D). Clinical outcomes in cases of NCSE could be related to the degree of structural brain damage, which may depend on the severity of neuronal injury caused by epileptic discharges. In case 2 , the extensive and marked decreased in IMZ uptake may be predictive 
Miwa K (2019) Utility of 123I-iomazenil single-photon emission computed tomography as an indicator of neurological irreversibility in patients with non-convulsive status epilepticus

of a poor treatment response and worsened outcome compared to patients with sparse and mild reductions in IMZ uptake.

To the best of our knowledge, no clinical study has reported on the regional distribution of IMZ uptake in cases of NCSE, although very few investigators have examined IMZ-SPECT findings in cases with mesial temporal lobe epilepsy. These few studies all concluded that IMZ-SPECT was useful for identifying epileptogenic foci that could not be detected by MRI [10-12]. Here, using IMZ-SPECT, we demonstrated considerably different findings in two cases with NCSE of unknown etiology: an extensive and marked decrease in IMZ uptake compared to a sparse and mild decrease in IMZ uptake. The patient with mild reductions recovered well, while the patient with more marked reductions had a poor prognosis.

In epilepsy, the application of IMP-SPECT imaging is based on the assumption that the increased ictal neuronal activity is associated with increased CBF. Neither IMP-SPECT nor MRI could identify the reversibility in neuronal function; however, IMZ-SPECT seemed predictive of the clinical outcomes in these patients with NCSE.

\section{Conclusion}

In the two NCSE patients presented in this report, IMZ-SPECT revealed different patterns of regional tracer accumulation: extensive and marked versus sparse and mild reductions in IMZ uptake. The former type is strongly suggestive of irreversible neuronal damage. Although our case reports are merely small examples, prospective studies are warranted to meet the lack of data of IMZ-SPECT and to shed more light on the potential role in clinical practice.

\section{Ethical statement}

The authors ensure that the work described has been carried out in accordance with The Code of Ethics of the World Medical Association (Declaration of Helsinki) for experiments involving humans.

\section{Consent}

Informed consent was obtained for experimentation with human subjects. The privacy rights of human subjects could always be observed

\section{Conflicts of interest}

The authors have declared that no competing interests exist.

\section{References}

1. Drislane FW (2000) Presentation, evaluation, and treatment of nonconvulsive status epilepticus. Epilepsy Behav 1: 301-314.

2. Bauer G, Trinka E (2010) Nonconvulsive status epilepticus and coma. Epilepsia 51: 177-190. [Crossref]

3. Leitinger M, Trinka E, Gardella E, et al. (2016) Diagnostic accuracy of the Salzburg EEG criteria for non-convulsive status epilepticus: A retrospective study. Lancet Neurol 15: 1054-1062.

4. Shuke N, Hashizume K, Kiriyama K, Okizaki A, Yamamoto W, et al. (2004) Correct localization of epileptogenic focus with I-123 iomazenil cerebral benzodiazepine receptor imaging: A case report of temporal lobe epilepsy with discordant ictal cerebral blood flow SPECT. Ann Nucl Med 18: 541-545. [Crossref]

5. Morimoto K, Tamagami H, Matsuda K (2005) Central-type benzodiazepine receptors and epileptogenesis: Basic mechanisms and clinical validity. Epilepsia 46: 184-188.

6. Umeoka S, Matsuda M, Baba K (2007) Usefulness of 123I-iomazenil SPECT to discriminate between mesial and lateral temporal lobe epilepsy in patients with normal MRI findings. $J$ Neurosurg 107: 352-363.

7. Van Huffelen AC, van Isselt JW, van Veelen CWM (1990) Identification of the side of epileptic focus with 123I-iomazenil SPECT: A comparison with 18FDG-PET and ictal EEG findings in patients with medically intractable complex partial seizures. Acta Neurochir Suppl (Wien) 50: 95-99.

8. Bartenstein P, Ludolph A, Schober O (1991) Benzodiazepine receptors and cerebral blood flow in partial epilepsy. Eur J Nucl Med 18: 111-118.

9. Cordes M, Henkes H, Ferstl F, Schmitz B, Hierholzer J, et al. (1992) Evaluation of focal epilepsy: A SPECT scanning comparison of 123-I-iomazenil versus HM-PAO. AJNR Am J Neuroradiol 13: 249-253. [Crossref]

10. Gainza-Lein M, Fernandez IS, Ulate-Campos A (2018) Timing in the treatment of status epilepticus: From basics to the clinic. Seizure. [Crossref]

11. Lamusuo S, Ruottinen HM, Knuuti J (1997) Comparison of [18F]-FDG-PET, [99mTc]HMPAO-SPECT, and [123I]-iomazenil-SPECT in localizing the epileptogenic cortex J Neurol Neurosurg Psychiatry 63: 743-748.

12. Sjoholm H, Rosen I, Elmqvist D (1995) Role of I-123-iomazenil SPECT imaging in drug resistant epilepsy with complex partial seizures. Acta Neurol Scand 92: 41-48.

Copyright: (C2019 Miwa K. This is an open-access article distributed under the terms of the Creative Commons Attribution License, which permits unrestricted use, distribution, and reproduction in any medium, provided the original author and source are credited. 